\title{
On trees with unique locating kernels
}

\section{Dorota Bród ${ }^{1}$}

Received: 15 April 2021 / Accepted: 7 July 2021 / Published online: 2 August 2021

(C) The Author(s) 2021

\begin{abstract}
A locating-dominating set of a graph $G$ is a set $D$ of vertices such that for every two vertices $x, y \in V(G) \backslash D$ the sets $N(x) \cap D$ and $N(y) \cap D$ are non-empty and different. In this paper, we define the locating kernel of a graph $G$, i.e., a subset of its vertex set which is independent and a locating-dominating set. We provide a constructive characterization of trees with a unique locating kernel.
\end{abstract}

Keywords Location-domination in graphs · Independence · Locating kernels $\cdot$ Characterization of structure $\cdot$ Tree

Mathematic subject classification 11B37 · 05C69

\section{Introduction}

In general, we use the standard terminology and notation of graph theory, cf. West [11]. Let $G$ be an undirected and a simple graph with vertex set $V(G)$ and edge set $E(G)$. The open neighborhood of a vertex $x \in V(G)$ is $N(x)=\{y \in V(G): x y \in E(G)\}$, the closed neighborhood of a vertex $x$ is $N[x]=N(x) \cup\{x\}$. The degree of $x$, denoted by ${ }_{G}^{\circ} x$, is the cardinality of the set $N(x)$. Let $T$ be a tree. A vertex of degree 1 is a leaf. Its neighbor is called a support vertex. Denote by $L(x)$ the set of leaves of $T$ attached to a support vertex $x$. If $|L(x)|=1(|L(x)| \geq 2$ ), then $x$ is called a weak support (strong support) vertex of $T$, respectively. By a branch vertex we mean a vertex of degree at least 3 . The set of branch vertices will be denoted by $B(T)$. Let $x \in B(T)$. By a branch at a vertex $x$ we mean a maximal subtree $B_{x}$ which includes $x$ and exactly one edge incident to $x$. A twig in $T$ is a nontrivial path joining a leaf of $T$ to the closest vertex in $T$ which is not

Dorota Bród

dorotab@prz.edu.pl

1 The Faculty of Mathematics and Applied Physics, Rzeszow University of Technology, al. Powstańców Warszawy 12, 35-959 Rzeszów, Poland 
of degree 2 . We denote by $P_{n}, K_{n}, C_{m}$ the $n$-vertex path, $n$-vertex complete graph, $m$ vertex cycle,/queryPlease check and provide caption for Table 1, 2, 3, amend if necessary. respectively, $n \geq 1, m \geq 3$. By a $\operatorname{star} K_{1, n-1}, n \geq 3$, we mean a connected graph in which exactly one vertex has degree greater than 1.

In a graph $G$, an independent set is a subset $S$ of $V(G)$ such that no two vertices in $S$ are adjacent. Moreover, an empty set and a subset of $V(G)$ containing only one vertex are independent. A set $D \subseteq V(G)$ is a dominating set of $G$ if every vertex of $V(G) \backslash D$ has a neighbor in $D$. A subset $J \subseteq V(G)$ which is independent and a dominating set is a kernel of $G$. In the literature, there are some variants and generalizations of kernels, see, e.g. [5, 12, 13]. A set $D \subseteq V(G)$ is a locatingdominating set if it is dominating and for every two vertices $x, y$ of $V(G) \backslash D$ holds $N(x) \cap D \neq N(y) \cap D$. Location-domination was introduced by Slater [8,9]. For recent studies on locating-dominating sets, we cite $[2,3]$. In this paper, we define a new kind of kernel in graphs using the concept of independence and locationdomination. By a locating kernel of a graph $G$ we mean such subset $J \subset V(G)$ that $J$ is independent and locating-dominating set. Clearly, a locating kernel of $G$ is a kernel of the graph in classical sense.

Consider a communication network with a set of broadcasting stations, which are able to send a message to itself and all his neighbors. To prevent interference from broadcasting stations, they are not adjacent. Moreover, any two stations can not receive messages from the same set of broadcasting stations, so that in the event of failure of all broadcasting stations from this set, at least one of them could receive a message from another broadcasting station. This situation can be modelled as a graph with vertices representing by stations and edges showing where is possible to construct lines of communications between stations. The problem of selecting a set of broadcasting stations becomes the problem of finding a locating kernel of the graph.

Not all graphs have a locating kernel. For example, the graphs $C_{4}, K_{n}, n \geq 3$ do not possess a locating kernel. The literature includes many papers dealing with the theory of independent sets, dominating sets and kernels. There are several papers devoted to characterizing graphs with maximal or minimal numbers of specified substructures. Recursive characterization of trees with some extremal domination properties has recently been considered in a number of papers, see, e.g. Henning [7], Haynes and Henning [6], Fischermann and Volkmann [4].

In [1], a characterization of trees with unique minimum locating-dominating sets was presented. The main goal of this paper is the characterization of trees with unique locating kernels.

It is easily seen that every tree has a locating kernel. Let $J$ be a locating kernel of a tree $T$. A vertex $v \in V(T)$ is called J-avoided vertex if $v$ does not belong to any locating kernel of $T$.

Proposition 1 Let $J$ be a locating kernel of T. Then every strong support vertex $x$ is a J-avoided vertex. Moreover $L(x) \subseteq J$.

Proof Assume that $x$ is a strong support vertex of $T$ with $L(x)=\left\{z_{1}, z_{2}, \ldots, z_{k}\right\}$, $k \geq 2$. Let $J$ be a locating kernel of $T$. Suppose that $x \in J$, then $z_{i} \notin J$ for $i=$ $1,2, \ldots, k$ and $N\left(z_{1}\right) \cap J=N\left(z_{2}\right) \cap J=\ldots=N\left(z_{k}\right) \cap J=\{x\}$, a contradiction. 


\section{Counting of locating kernels}

Denote by $q(G)$ the number of locating kernels of a graph $G$. We start presenting general rules for evaluating $q(G)$. Let $x$ be any vertex of $G$. Denote by $q_{x}(G)$ the number of locating kernels of $G$ which all include the vertex $x$. On the other hand, let $q_{-x}(G)$ be the count of locating kernels in $G$ which all avoid the vertex $x, q_{-x}(G)$ being zero exactly if $x$ has no neighbor in $G$. Then the basic rule for recursively evaluating the number of locating kernels in a graph $G$ is as follows:

$$
q(G)=q_{x}(G)+q_{-x}(G) \quad \text { for any vertex } x \text { of } G .
$$

Theorem 1 If $H_{i}, \quad i=1,2, \ldots, k$ are connected components of $G$ then $q(G)=\prod_{i=1}^{k} q\left(H_{i}\right)$

Theorem 2 Let $T \neq K_{1, n-1}$ and let $x$ be a strong support vertex of $T$. Then $q(T)=q(T-x-L(x))$.

Using Proposition 1 and Theorems 1 and 2, we obtain the next result.

Theorem 3 Let $T$ be a tree with a strong support vertex $x$ of degree $b \geq 3$ and with $b$ branches $B_{x}^{i}$ at $x$. Then

$$
q(T)=\prod_{i=1}^{b} q\left(B_{x}^{i}-x\right)
$$

Next we give the recurrence formula for the number of locating kernels of $n$ vertex path. We assume that vertices of $P_{n}$ are numbered in the natural fashion.

Theorem 4 Let $P_{n}$ be a path on $n$ vertices, $n \geq 1$. Then

$$
q\left(P_{n}\right)=q\left(P_{n-2}\right)+q\left(P_{n-5}\right) \text { for } n \geq 6
$$

with initial conditions $q\left(P_{1}\right)=q\left(P_{3}\right)=1, q\left(P_{2}\right)=q\left(P_{5}\right)=2, q\left(P_{4}\right)=3$.

Proof The initial conditions are obvious. It is easy to check that $q\left(P_{6}\right)=q\left(P_{7}\right)=4$. Let $n \geq 8$. Assume that $J$ is a locating kernel of $P_{n}$. Consider two cases.

Case 1: $x_{n} \in J$.

Then $x_{n-1} \notin J$. We have two possibilities.

1.1: $\quad x_{n-2} \in J$.

Let $\mathcal{J}_{1}$ be a family of locating kernels $J$ of $P_{n}$ such that $x_{n}, x_{n-2} \in J$. Then $x_{n-3} \notin J$. Hence $J=J^{\prime} \cup\left\{x_{n}, x_{n-2}\right\}$, where $J^{\prime}$ is any locating kernel of $P_{n}^{\prime}=P_{n}-\left\{x_{n}, x_{n-1}, x_{n-2}, x_{n-3}\right\}$, which is isomorphic to $P_{n-4}$. Thus, $\left|\mathcal{J}_{1}\right|=q\left(P_{n-4}\right)$. 


\section{2: $\quad x_{n-3} \in J$.}

Let $\mathcal{J}_{2}$ be a family of locating kernels $J$ of $P_{n}$ such that $x_{n}, x_{n-3} \in J$. Then $x_{n-5} \in J$ and $x_{n-6} \notin J$. Hence $J=J^{\prime \prime} \cup\left\{x_{n}, x_{n-3}, x_{n-5}\right\}$, where $J^{\prime \prime}$ is any locating kernel of $P_{n}^{\prime \prime}=P_{n}-\left\{x_{n-i} \mid i=0,1, \ldots, 6\right\}$, which is isomorphic to $P_{n-7}$. Thus, $\left|\mathcal{J}_{2}\right|=q\left(P_{n-7}\right)$.

Case 2: $\quad x_{n} \notin J$.

Let $\mathcal{J}_{3}$ be a family of locating kernels $J$ of $P_{n}$ such that $x_{n} \notin J$. Then $x_{n-1} \in J$. Suppose that $x_{n-4} \in J$. Then $N\left(x_{n-2}\right) \cap J=\left\{x_{n-1}\right\}=N\left(x_{n}\right) \cap J$, a contradiction. Hence, we have $x_{n-3} \in J$. Proving analogously as in Case 1.1 we obtain $\left|\mathcal{J}_{3}\right|=q\left(P_{n-5}\right)$.

Consequently $q\left(P_{n}\right)=q\left(P_{n-4}\right)+q\left(P_{n-5}\right)+q\left(P_{n-7}\right)$ for $n \geq 8$.

By simple calculations, we have

$$
q\left(P_{n}\right)=q\left(P_{n-2}\right)+q\left(P_{n-5}\right)=q\left(P_{n-4}\right)+q\left(P_{n-7}\right)+q\left(P_{n-5}\right),
$$

which ends the proof.

Remark 1 It is worth mentioning that $q\left(P_{n}\right)=a_{n+6}$, where the sequence $\left\{a_{n}\right\}$ defined as $a_{n}=a_{n-2}+a_{n-5}$ for $n \geq 5, a_{0}=a_{2}=a_{4}=0, a_{1}=a_{3}=a_{5}=a_{6}=1$, is the sequence A001687 in [10].

The number of locating kernels of the graph $P_{n}, n \geq 3$, (for $3 \leq n \leq 15$, see Table 1) has the following combinatorial interpretation: $q\left(P_{n}\right)$ is the number of ways of writing $n-1 \quad\left(n-1=\left|E\left(P_{n}\right)\right|\right)$ as an ordered sum of $i \geq 1$ terms $a_{1}, a_{2}, \ldots, a_{i}$ such that $a_{1}, a_{i} \in\{1,2,3\}$ and $a_{j} \in\{2,3\}$ for $j \neq 1, i$, and if $a_{j}=3$ for some $j \in\{1, \ldots, i\}$, then $a_{j-1}=a_{j+1}=2$ (if such exist). For example, by Table 1 , $q\left(P_{11}\right)=11$. There are 11 compositions of 10 that satisfy the assumptions above. They are presented in Table 2.

\section{Minimal trees}

Let $n$ and $d$ be integers such that $d \geq 3$ and $n \geq d+1$. The palm $P_{n, d}$ is the graph obtained from two vertex disjoint graphs: a star $K_{1, d-1}$ and a path $P_{n-d}$ by adding an edge joining the center vertex of $K_{1, d-1}$ to an end vertex of $P_{n-d}$. If $n=d+1$, then $P_{n, n-1}=K_{1, n-1}$. Using Theorem 3 we have $q\left(K_{1, n-1}\right)=1$ for $n \geq 3$. By Theorem 2 we obtain the next result.

Table 1 The number of locating kernels of $n$-vertex path

\begin{tabular}{lllllllllllllllll}
\hline$n$ & 1 & 2 & 3 & 4 & 5 & 6 & 7 & 8 & 9 & 10 & 11 & 12 & 13 & 14 & 15 \\
\hline$q\left(P_{n}\right)$ & 1 & 2 & 1 & 3 & 2 & 4 & 4 & 5 & 7 & 7 & 11 & 11 & 16 & 18 & 23 \\
\hline
\end{tabular}


Table 2 A combinatorial interpretation of the number of locating kernels of path on 11 vertices

\begin{tabular}{ll}
\hline Locating kernel of $P_{11}$ & Composition of 10 \\
\hline$J_{1}=\left\{x_{1}, x_{3}, x_{5}, x_{7}, x_{9}, x_{11}\right\}$ & $2+2+2+2+2$ \\
$J_{2}=\left\{x_{2}, x_{4}, x_{6}, x_{8}, x_{10}\right\}$ & $1+2+2+2+2+1$ \\
$J_{3}=\left\{x_{1}, x_{4}, x_{6}, x_{8}, x_{11}\right\}$ & $3+2+2+3$ \\
$J_{4}=\left\{x_{1}, x_{4}, x_{6}, x_{9}, x_{11}\right\}$ & $3+2+3+2$ \\
$J_{5}=\left\{x_{1}, x_{3}, x_{6}, x_{8}, x_{11}\right\}$ & $2+3+2+3$ \\
$J_{6}=\left\{x_{2}, x_{4}, x_{6}, x_{8}, x_{11}\right\}$ & $1+2+2+2+3$ \\
$J_{7}=\left\{x_{1}, x_{4}, x_{6}, x_{8}, x_{10}\right\}$ & $3+2+2+2+1$ \\
$J_{8}=\left\{x_{2}, x_{4}, x_{7}, x_{9}, x_{11}\right\}$ & $1+2+3+2+2$ \\
$J_{9}=\left\{x_{1}, x_{3}, x_{5}, x_{8}, x_{10}\right\}$ & $2+2+3+2+1$ \\
$J_{10}=\left\{x_{2}, x_{4}, x_{6}, x_{9}, x_{11}\right\}$ & $1+2+2+3+2$ \\
$J_{11}=\left\{x_{1}, x_{3}, x_{6}, x_{8}, x_{10}\right\}$ & $2+3+2+2+1$ \\
\hline
\end{tabular}

Proposition 2 Let $n \geq 4, d \geq 3$ be integers. Then $q\left(P_{n, d}\right)=q\left(P_{n-d}\right)$. In particular, $q\left(P_{d+3, d}\right)=1$.

Let $r \geq 2, d_{i} \geq 2, i=1,2, \ldots, r$. By an $r$-star we mean a tree $S_{d_{1}, d_{2}, \ldots, d_{r}}$ with $r+\sum_{i=1}^{r} d_{i}$ vertices such that $r$ vertices $v_{1}, v_{2}, \ldots, v_{r}$ have degree $d_{1}+1, d_{2}+2, d_{3}+2, \ldots, d_{r-1}+2, d_{r}+1$, respectively, the remaining vertices have degree one and every vertex $v_{i}$ is adjacent to $v_{i+1}, i=1,2, \ldots, r-1$. For $r=1$, we assume that $S_{d_{1}}=K_{1, d_{1}}$. By Theorem 2, we get $q\left(S_{d_{1}, d_{2}, \ldots, d_{r}}\right)=1$.

A tree $T$ is minimal, if $q(T)=1$. Let $\mathcal{T}_{n}^{\min }$ be the class of $n$-vertex minimal trees. It is easy to check that for $n=1,3,4,5$ there exists exactly one minimal tree. For $n=2$, there does not exist a minimal tree. All minimal trees of order up to 10 have been found by inspection. Numbers of those trees are given in Table 3 . All $n$-vertex minimal trees for $1 \leq n \leq 9$ and $n \neq 2$ are presented in Fig. 1 .

We begin by giving useful observations. It is easy to see that if $T$ is an $n$-vertex minimal tree and $n \geq 4$, then $T \neq P_{n}$.

Proposition 3 Let $T_{1}, T_{2}$ be trees with strong support vertices $x_{1}, x_{2}$, respectively. Let $P_{k}$ be a path on $k$ vertices, $k=2$ or $k \geq 4$, with endvertices $v_{1}, v_{k}$. Assume that $T^{\prime}$ is a tree obtained from trees $T_{1}, T_{2}$ and $P_{k}$ by adding edges $x_{1} v_{1}$ and $x_{2} v_{k}$. Then $T^{\prime}$ is not a minimal tree.

Proof Since $x_{1}$ and $x_{2}$ are strong support vertices of $T^{\prime}$, by Theorem 2, we get

Table 3 The number of minimal trees of order up to 10

\begin{tabular}{lllllllllll}
\hline$n$ & 1 & 2 & 3 & 4 & 5 & 6 & 7 & 8 & 9 & 10 \\
\hline$\left|\mathcal{T}_{n}^{\text {min }}\right|$ & 1 & 0 & 1 & 1 & 1 & 3 & 4 & 5 & 13 & 22 \\
\hline
\end{tabular}




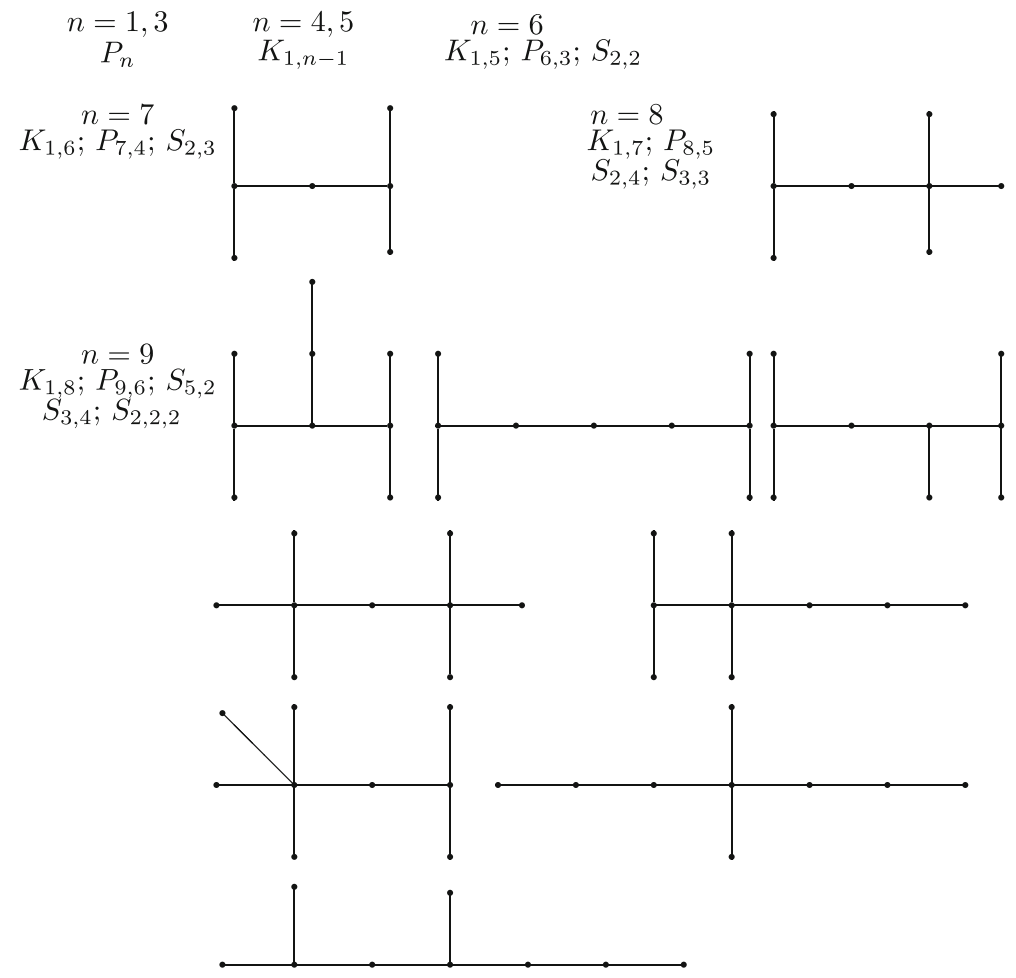

Fig. 1 All $n$-vertex minimal trees, $1 \leq n \leq 9, n \neq 2$

$$
q\left(T^{\prime}\right)=q\left(T^{\prime}-x_{1}-L\left(x_{1}\right)-x_{2}-L\left(x_{2}\right)\right) .
$$

For every connected component $T_{i}$ of the tree $T^{\prime}-x_{1}-L\left(x_{1}\right)-x_{2}-L\left(x_{2}\right)$, we have $q\left(T_{i}\right) \geq 1$. Because a path $P_{k}(k=2$ or $k \geq 4)$ is one of the connected components, by Theorems 1 and 4 , we get $q\left(T^{\prime}\right) \geq q\left(P_{k}\right) \geq 2$. If all neighbors of $x_{1}$ and $x_{2}$ are leaves in $T_{1}, T_{2}$, respectively, then, by Theorem $3, q\left(T^{\prime}\right)=q\left(P_{k}\right) \geq 2$.

In the same way, we can prove the next Propositions.

Proposition 4 Let $T$ be a minimal tree with a strong support vertex $x$. Then there is no twig of length $k, k=2$ or $k \geq 4$, attached to $x$.

Proposition 5 Let $x$ be non-support branch vertex of $T$ with twig of length at least 3. Then $T$ is not a minimal tree.

Proposition 6 Let $T$ be a minimal tree with a branch vertex $x$. Then there is no twig of length $k, k \geq 4$, attached to $x$.

Proof Let $x$ be a branch vertex with a twig of length 4 of a minimal tree $T$ and let $J$ be a locating kernel of $T$. If $x$ is a strong support vertex, then the desired result holds by Proposition 4. Assume that $x$ is not a strong support vertex. Let $z$ be the leaf on the twig and $y$ be the neighbor of $z, v$ be the neighbor of degree 2 of $x$ and let $u$ be 
the neighbor of $y$ and $v$. Then $x$ can be an element of $J$. Assume that $x \in J$. Then $z, u \in J$. Moreover, there exists an additional locating kernel $J^{\prime}$ such that $x \notin J^{\prime}$ (then $y, v \in J^{\prime}$ ), which contradicts the minimality of $T$.

In the similar way, we can prove the desired result for $k \geq 5$.

Denote by $L(T)$ the set of leaves of a tree $T$.

Proposition 7 Let $T$ be an $n$-vertex minimal tree, $n \geq 3$, with a unique locating kernel J. Then $L(T) \subseteq J$.

Proof It is easily seen that if $T=S_{d_{1}, d_{2}, \ldots, d_{r}}$, then $J=L\left(S_{d_{1}, d_{2}, \ldots, d_{r}}\right)$. Assume that $T$ is a minimal tree and is not an $r$-star, $r \geq 1$. Let $y$ be a strong support vertex of $T$. Then by Proposition $1, L(y) \subset J$. Assume now that $y$ is a weak support vertex of $T$ with $L(y)=\{z\}$. Suppose that $z \notin J$. Then $y \in J$. Consider the following possibilities:

Case 1: ${ }^{\circ} y=2$.

1.1: $\quad{ }^{\circ} w=2$.

Let $w$ be a neighbor of $y, w \neq z$.

Let $v$ be the second neighbor of $w,(v \neq y)$. Suppose that ${ }^{\circ} T=2$. Then there exists a branch vertex $u$, the neighbor of $v$, with a twig of length 4 , a contradiction (Proposition 6). Assume now that ${ }^{\circ} v \geq 3$. If $v$ is a strong support vertex then, by Proposition $1, v$ is $J$-avoided and $N(w) \cap J=N(z) \cap J=\{y\}$, a contradiction. Assume now that $v$ is not a strong support vertex and $N(v)=\left\{w, u_{1}, u_{2}, \ldots, u_{k}\right\}, k \geq 2$. Then we have two possibilities: $v \in J$ or $v \notin J$. In the second case, we have multiple choices $u_{i} \in J$ and all the choices produce a locating kernel, a contradiction.

1.2: $\quad{ }^{\circ} w \geq 3$.

Let $N(w)=\left\{y, v_{1}, v_{2}, \ldots, v_{j}\right\}, j \geq 2$. Then every nonempty subset of the set $N(w) \backslash\{y\}$ is the subset of $J$. Thus all the choices of such subsets of $N(w) \backslash\{y\}$ produce a locating kernel of $T$, a contradiction with the minimality of $T$. In case if all vertices $v_{j}$ are strong support vertices, we have $N(w) \cap J=N(z) \cap J=\{y\}$, a contradiction.

Case 2: $\quad{ }_{T}^{\circ} y \geq 3$.

Consider the following subcases.

2.1: $\quad y$ has a neighbor, say $w$, of degree 2 .

Consider two possibilities.

2.1.1: $\quad w$ is adjacent to a vertex, say $p$, of degree 2 .

Then, by Proposition 6,w is on a twig of length 3 or there exists the second neighbor of $p$, of degree 2 , which is adjacent to a support vertex. Assume that $w$ is on the twig of length 3. Let $z_{1}$ be a leaf adjacent to $p$. Because $y \in J$, we have that either $p \in J$ or $z_{1} \in J$, a contradiction with the minimality of $T$. Assume now that $s, s \neq w$, is the neighbor of $p$ of degree 2 and $s$ is adjacent to a support vertex. Then either $p \in J$ or $s \in J$, a contradiction with the minimality of $T$. 
2.1.2: $w$ is adjacent to a branch vertex $x, x \neq y$.

If $x$ is a strong support vertex, then $x$ is $J$-avoided and $N(w) \cap J=N(z) \cap J=\{y\}$, a contradiction. If $x$ is not a strong support vertex, then either $x \in J$ or $x$ is locally dominated by at least one of its neighbors, a contradiction.

2.2: $\quad y$ has all neighbors, except of $z$, of degree at least 3 .

Let $v_{1}, v_{2}, \ldots, v_{k}, k \geq 2$, be neighbors of $y$. Assume that none of neighbors of $y$ is a strong support vertex. Let $N\left(v_{1}\right)=\left\{u_{1}, u_{2}, \ldots, u_{k}\right\}$, $N\left(v_{2}\right)=\left\{w_{1}, w_{2}, \ldots, w_{l}\right\}, k, l \geq 2$. Because $y \in J$, we have multiple choice $u_{i} \in J$ and $w_{j} \in J, i=1,2, \ldots, k, j=1,2, \ldots, l$, a contradiction. Similarly we get a contradiction in the case if $v_{1}$ is a strong support vertex and $v_{2}$ is not a support vertex. It is easily seen that in case if all neighbors of $y$, except of $z$, are strong support vertex, then $T$ is not a minimal tree.

Let $T$ be a minimal $n$-vertex tree, $n \geq 3$. Recall that $L(T)$ is the set of leaves of $T$. We will use the following notation:

$S(T)$ - the set of support vertices of $T$,

$N(T)$ - the set of neighbors of support vertices of $T$ not being leaves and support vertices,

$R(T) \quad$ - the set of the remaining vertices of $T$, i.e. $R(T)=V(T) \backslash(L(T) \cup N(T) \cup S(T))$.

It is easily seen that the sets $L(T), S(T), N(T)$ and $R(T)$ are disjoint sets. In case if $N(T)=\emptyset$ and $R(T)=\emptyset$, then $V(T)=L(T) \cup S(T)$ and $T$ is isomorphic to an $r$-star, $r \geq 1$.

Let $x \in V(T)$. We define the status of $x$, denoted by $\operatorname{sta}(x)$, to be $L, S, N, R$. If $x \in L(T)$ then $\operatorname{sta}(x)=L$, if $x \in S(T)$ then $\operatorname{sta}(x)=S$, if $x \in N(T)$ then $\operatorname{sta}(x)=N$, if $x \in R(T)$ then $\operatorname{sta}(x)=R$. Let $J$ be a unique locating kernel of $T$. Then, by Proposition 7, we have that if $\operatorname{sta}(x)=L$ then $x \in J$. Moreover, by Proposition 7, if $\operatorname{sta}(x)=S$ then $x \notin J$.

Proposition 8 Let $T$ be $n$-vertex minimal tree $T$ with $x \in R(T)$. Then $n \geq 9$.

Remark 2 It is easy to observe that if $T$ is a minimal tree with a unique locating kernel $J$ and $x \in N(T)$ then $x$ can be element of $J$ or can be $J$-avoided. For example, if $T$ is a tree obtained from $r$-star $S_{d_{1}, d_{2}, \ldots, d_{r}}, r \geq 2$, by inserting a new vertex on some edges joining vertices $v_{i}, v_{i+1}, 1 \leq i \leq r-1$, then for every $x \in N(T)$ holds $x \in J$.

The next lemma gives sufficient and necessary conditions for minimal trees $T$ with a $J$-avoided vertex $x \in N(T)$.

Lemma 1 Let $T$ be a minimal tree with a unique locating kernel J. Assume that $x \in N(T)$ and $u$ is a support vertex adjacent to $x$. Then $x$ is J-avoided if and only if there exist at least two neighbors $w_{1}, w_{2}$ of $x,\left(w_{i} \neq u, i=1,2\right)$ such that every neighbour of $w_{i}$ (except of $x$ ) is a support vertex.

Proof Assume that $T$ is a minimal tree with a unique locating kernel $J$. Let $w_{1}, w_{2}$ be two neighbors of $x, w_{i} \neq u, i=1,2$. Assume that vertices $w_{1}, w_{2}$ are adjacent to 
support vertices, say $u_{1}, u_{2}$, respectively. By Proposition 7 vertices $u_{1}, u_{2}$ are $J$ avoided. Suppose that $x \in J$. Then $w_{1}, w_{2} \notin J$ and $N\left(w_{1}\right) \cap J=N\left(w_{2}\right) \cap J=\{x\}$, a contradiction. Thus, $x$ is $J$-avoided.

Assume now that $x$ is $J$-avoided. Consider the following cases.

Case 1: ${ }^{\circ} x=2$.

1.1: $\quad w \in S(T)$.

$$
\text { Let } N(x)=\{u, w\} \text {. }
$$

Then $w$ is $J$-avoided, hence $x \in J$, a contradiction.

1.2: $\quad w \notin S(T)$.

Then either $x \in J$ or $w \in J$, a contradiction with the minimality of $T$.

Case 2: $\quad{ }_{T}^{\circ} x \geq 3$ and there exists only one neighbour of $x$, say $w_{1}, w_{1} \neq u$, which is adjacent to a strong vertex. Let $w$ be a neighbour of $x, w \neq w_{1}, w \neq u$. Consider the following possibilities:

2.1: $\quad{ }^{\circ} w=2$.

2.1.1: $\quad w$ is on the twig of length $k$ at $x$.

By Proposition 6, we have $k=2$ or $k=3$. If $k=2$, then $w$ is a weak support vertex. Let $N(w)=\{x, p\}$. Then either $w \in J$ or $p \in J$, a contradiction.

Let $k=3$ and $x-w-q-z$ be the twig at $x$. Then either $w, z \in J$ or $x, q \in J$, a contradiction.

2.1.2: $\quad$ There exists a path $x-w-t-\cdots-y$ of length $m$, such that $y \in B(T)$. Let $m=2$. Then $t \in B(T)$. If $t$ is a weak support vertex then either $w \in J$ or $x \in J$, a contradiction. Analogously we can prove that $T$ is not a minimal tree if ${ }^{\circ} t \geq 3$ and $t \notin S(T)$. Let $m \geq 3$. Assume that $y \in S(T)$. Then $y \notin J$ and either $w \in J$ or $t \in J$, a contradiction. Let $y \notin S(T)$. Then either $w, y \in J$ or $x, t \in J$, a contradiction.

2.2: $\quad{ }^{\circ} w \geq 3$.

If $w \in S(T)$ then $w$ is $J$-avoided and either $x \in J$ or $w_{1} \in J$, a contradiction. Let $w \notin S(T)$.

2.2.1: Assume that there is a twig of length $l$ at $w$. By Proposition 6, we have $l=2$ or $l=3$. If $l=2$ and $w-v-s$ be the twig. Then either $w, s \in J$ or $x, s \in J$, a contradiction. Analogously, we can prove that if $l=3$ then $T$ is not a minimal tree.

2.2.2: $\quad$ There exists a vertex $y_{m} \in B(T)$ such that $w$ is on the path $w-y_{1}-$ $y_{2}-\cdots-y_{m}$ of length $m$. Let $m=1$. Then $y_{1} \in B(T) \backslash S(T)$. Then either $y_{1} \in J$ or $w \in J$, which contradicts the minimality of $T$. Let $m \geq 2$. Then either $y_{m} \in J$ or $y_{m} \notin J$, a contradiction.

Case 3: ${ }^{\circ} x \geq 3$ and $x$ is not adjacent to a vertex which is adjacent to a support vertex.

3.1: $\quad$ All of neighbors of $x$ are support vertices. Then $x \in J$.

3.2: $\quad$ There exists at least one neighbor of $x$ of degree at least 3 , say $t$, such that $t \notin S(T)$. Then either $x \in J$ or $t \in J$, a contradiction. 
Now we describe a procedure for building a family of trees with unique locating kernels. These trees can be obtained recursively as follows:

Let $T_{1}, T_{2}$ be two vertex-disjoint minimal trees, each of order at least 3 , with unique locating kernels $J_{1}, J_{2}$, respectively. We define four operations that can be used for linking trees $T_{1}$ and $T_{2}$ by an edge and producing a new minimal tree.

Operation $\mathcal{O}_{1}$ : Let $T$ be the tree obtained from $T_{1}$ and $T_{2}$ by adding an edge joining a vertex $x \in V\left(T_{1}\right)$ and $y \in V\left(T_{2}\right)$ such that $\operatorname{sta}(x) \in\{L, N\}$ and $\operatorname{sta}(y) \in$ $\{S, R\}$ and if $\operatorname{sta}(y)=R$, then $y$ is $J_{2}$-avoided.

Operation $\mathcal{O}_{2}$ : Let $T$ be the tree obtained from $T_{1}$ and $T_{2}$ by adding an edge joining a vertex $x \in V\left(T_{1}\right)$ and $y \in V\left(T_{2}\right)$ such that

(a) $\operatorname{sta}(x)=\operatorname{sta}(y)=S$ or

(b) $\operatorname{sta}(x)=S$ and $\operatorname{sta}(y)=R$ or

(c) $\operatorname{sta}(x)=\operatorname{sta}(y)=R$ and $x$ is $J_{1}$-avoided or $y$ is $J_{2}$-avoided.

Operation $\mathcal{O}_{3}$ : Let $T$ be the tree obtained from $T_{1}$ and $T_{2}$ by adding an edge joining a vertex $x \in V\left(T_{1}\right)$ and $y \in V\left(T_{2}\right)$ such that $\operatorname{sta}(x)=\operatorname{sta}(y)=N$ and $x$ is $J_{1}$-avoided or $y$ is $J_{2}$-avoided.

Operation $\mathcal{O}_{4}$ : Let $T$ be the tree obtained from $T_{1}$ and $T_{2}$ by adding an edge joining a vertex $x \in V\left(T_{1}\right)$ and $y \in V\left(T_{2}\right)$ such that $\operatorname{sta}(x)=L, \operatorname{sta}(y)=N$ and $y$ is $J_{2}$-avoided.

Theorem 5 Let $T_{1}, T_{2}$ be two vertex-disjoint minimal trees, each of order at least 3 , with unique locating kernels $J_{1}, J_{2}$, respectively. Then the tree $T$ obtained from $T_{1}$ and $T_{2}$ by performing operation $\mathcal{O}_{1}, \mathcal{O}_{2}, \mathcal{O}_{3}$ or $\mathcal{O}_{4}$ is a minimal tree. Moreover, $J_{1} \cup J_{2}$ is the unique locating kernel of $T$.

Proof Let $x \in V\left(T_{1}\right), y \in V\left(T_{2}\right)$ and let $J_{1}, J_{2}$ be the unique locating kernels of $T_{1}$, $T_{2}$, respectively.

Case 1: $T$ is produced by an operation $\mathcal{O}_{1}$. Consider four cases.

1.1: $\quad \operatorname{sta}(x)=L$ and $\operatorname{sta}(y)=S$.

Then $x \in J_{1}$ and $y \notin J_{2}$, hence the set $J=J_{1} \cup J_{2}$ is a locating kernel of $T$. Assume that $J^{\prime}$ is an extra locating kernel of $T$. Consider the following possibilities.

1.1.1: $\quad x \notin J^{\prime}$ and $y \in J^{\prime}$.

Because $\operatorname{sta}(y)=S$ in $T$, by Proposition 7, we have that $y$ is $J^{\prime}$-avoided, a contradiction. 
1.1.2: $\quad x, y \notin J^{\prime}$.

Let $w$ be the neighbor of $x$ in $T_{1}$. Then $w \in J^{\prime}$. Consider the following possibilities:

(a) ${ }^{\circ} T_{1} w=2$.

Then $w$ is a weak support vertex of $T_{1}$, but is not a support vertex of $T$. By Proposition 6 we have that there exists in $T_{1}$ a branch vertex $v$ with a twig of length 2 or 3.

Assume that $w$ is adjacent to the branch vertex $v$. It easy to check that if $v \in S\left(T_{1}\right)$ then $T_{1}$ is not a minimal tree. Hence $v \notin S\left(T_{1}\right)$ and there exist at least two non-leaves neighbors of $v$, say $v_{1}, v_{2}, \ldots, v_{i}, i \geq 2$. Thus we have either $v, x \in J_{1}$ or we have multiple choices $v_{i} \in J_{1}$, a contradiction. In case if $v_{i} \in S\left(T_{1}\right)$ for every $i \geq 1$, then $N(v) \cap J^{\prime}=N(x) \cap J^{\prime}=\{w\}$, a contradiction.

Suppose now that there exists in $T_{1}$ a twig of length 3 , say $v-p-w-x$. Then, by Proposition 5, $v \in S(T)$ and $N(p) \cap J^{\prime}=N(x) \cap J^{\prime}=\{w\}$, a contradiction. (b) ${ }^{\circ} T_{1} w \geq 3$ and $w$ is a strong support vertex.

Then $w$ is $J^{\prime}$-avoided and $x$ is not locally dominated, a contradiction. (c) ${ }^{\circ} w_{T_{1}} \geq 3$ and $w$ is a weak support vertex.

Let $w_{1}, w_{2}, \ldots, w_{j}, w_{j} \neq x, j \geq 2$, be neighbors of $w$. Then at least one of vertices $w_{j}$, say $w_{1}$, is of degree 2 (otherwise $T_{1}$ is not a minimal tree). If $w_{1} \in N(T)$, then $N\left(w_{1}\right) \cap J^{\prime}=N(x) \cap J^{\prime}=\{w\}$, a contradiction. Suppose that $w_{1}$ is adjacent to a non-support branch vertex $s$. Then either $s \in J^{\prime}$ or $s \notin J^{\prime}$, a contradiction with the minimality of $T$. From above cases, we obtain that there is no a locating kernel $J^{\prime}$ such that $x, y \notin J^{\prime}$. Thus, there is no an extra locating kernel in this case and $T$ is a minimal tree.

1.2: $\quad \operatorname{sta}(x)=L, \operatorname{sta}(y)=R$ and $y$ is $J_{2}$-avoided.

Then $x \in J_{1}$ and $y \notin J_{2}$. Moreover, $J_{1} \cup J_{2}$ is a locating kernel of $T$.

Proving analogously as in Case 1.1 .2 we obtain that there is no a locating kernel $J^{\prime}$ of $T$ such that $x, y \notin J^{\prime}$. It is easily to check that there is no an additional locating kernel $J^{\prime \prime}$ of $T$ such that $x \notin J^{\prime \prime}$ and $y \in J^{\prime \prime}$.

1.3: $\quad \operatorname{sta}(x)=N$ and $\operatorname{sta}(y)=S$.

Then $y \notin J_{2}$ and $\operatorname{sta}(y)=S$ in $T$, too. Then the set $J=J_{1} \cup J_{2}$ is a locating kernel of $T$. Consider two possibilities.

(a) $x \in J_{1}$.

Assume that $J^{\prime}$ is a such locating kernel of $T$ that $x, y \notin J^{\prime}$. Suppose that there exists a neighbor of $x$ in $T_{1}$, say $v$, such that $v \notin S\left(T_{1}\right)$. Hence, $v \in R(T)$ or $v \in N(T)$ and ${ }^{\circ} T_{1} v \geq 2$. Then there is a vertex $w$ of degree 2 adjacent to $v$ and $w \in N(T)$ (otherwise, if ${ }^{\circ} T_{1} w \geq 3, T_{1}$ is not a minimal tree). Let $v \in J^{\prime}$, then $N(x) \cap J^{\prime}=N(w) \cap J^{\prime}=\{v\}$, a contradiction. In case, if all neighbors of $x$ are support vertices, $x$ is not locally dominated. (b) $x \notin J_{1}$.

Then, by Lemma 1 , there exist two neighbors $w_{i}, i=1,2$ of $x$, such that every vertex $w_{i}$, is adjacent to a support vertex. Then $\operatorname{sta}(x)=N$ in $T$, too. Assume that there is an extra locating kernel $J^{\prime \prime}$ of $T$ such that $x \in J^{\prime \prime}$. By Lemma $1, x$ is $J^{\prime \prime}$-avoided, a contradiction. 
1.4: $\quad \operatorname{sta}(x)=N, \operatorname{sta}(y)=R$ and $y$ is $J_{2}$-avoided.

Proving analogously as in Case 1.3 we obtain the result.

Case 2: $T$ is produced by an operation $\mathcal{O}_{2}$. Consider three cases.

2.1: $\quad \operatorname{sta}(x)=S$ and $\operatorname{sta}(y)=S$.

Then $x \notin J_{1}$ and $y \notin J_{2}$. It is easily seen that the set $J_{1} \cup J_{2}$ is a locating kernel of $T$. Because $\operatorname{sta}(x)=\operatorname{sta}(y)=S$ in $T$, we have by Proposition 7, that vertices $x$ and $y$ are not elements of any locating kernel of $T$. Hence, there is no an additional locating kernel in $T$.

2.2: $\quad \operatorname{sta}(x)=R$ and $\operatorname{sta}(y)=S$.

Then $\left(x \in J_{1}\right.$ or $\left.x \notin J_{1}\right)$ and $y \notin J_{2}$. Hence, in both cases the set $J_{1} \cup J_{2}$ is a locating kernel of $T$. Let $J^{\prime}$ be a locating kernel of $T$ such that $x \notin J^{\prime}$ and $y \in J^{\prime}$. By Proposition 7, $y$ is $J^{\prime}$-avoided, a contradiction.

2.3: $\quad \operatorname{sta}(x)=\operatorname{sta}(y)=R$ and $x$ is $J_{1}$-avoided or $y$ is $J_{2}$-avoided.

Assume that only $x$ is $J_{1}$-avoided. Then $x \notin J_{1}$ and $y \in J_{2}$. Hence, the set $J_{1} \cup J_{2}$ is a locating kernel of $T$. Assume that $J^{\prime}$ is a locating kernel of $T$ such that $x \in J^{\prime}$ and $y \notin J^{\prime}$. Then ${ }^{\circ} T x \geq 2$. Because $\operatorname{sta}(x)=R$ in $T_{1}$ and $x \in J_{1}$, we have that there exist two neighbors of $x$ in $T_{1}$, say $v_{1}, v_{2}$, which are attached to support vertices $w_{1}, w_{2}$, respectively. Thus $N\left(v_{1}\right) \cap J^{\prime}=N\left(v_{2}\right) \cap J^{\prime}=\{x\}$, a contradiction. It is easy to check that there is no any additional locating kernel $J^{\prime \prime}$ such that $x, y \notin J^{\prime \prime}$. The case when $x$ is $J_{1}$-avoided and $y$ is $J_{2}$-avoided we can prove in the similar way.

Case 3: $\quad T$ is produced by an operation $\mathcal{O}_{3}$. Consider two cases.

3.1: $\quad$ Let $\operatorname{sta}(x)=\operatorname{sta}(y)=N, x$ is $J_{1}$-avoided and $y \in J_{2}$.

Then the set $J_{1} \cup J_{2}$ is a locating kernel of $T$.

Suppose that $J^{\prime}$ is a locating kernel of $T$ such that $x, y \notin J^{\prime}$. Then, by Lemma 1 , we get a contradiction, because $y$ is not $J^{\prime}$-avoided.

3.2: $\quad$ Let $\operatorname{sta}(x)=\operatorname{sta}(y)=N, x$ is $J_{1}$-avoided and $y$ is $J_{2}$-avoided.

Then the set $J_{1} \cup J_{2}$ is a locating kernel of $T$. Assume that $x \in J^{\prime}$. Then, by Lemma 1 , there exist two neighbours of $x$, say $w_{1}, w_{2}$, such that every vertex $w_{i}$ is adjacent to a support vertex. Then

$N\left(w_{1}\right) \cap J^{\prime}=N\left(w_{2}\right) \cap J^{\prime}=\{x\}$, a contradiction. Similarly, we can prove that there is no a kernel $J^{\prime \prime}$ of $T$ such that $x \notin J^{\prime \prime}$ and $y \notin J^{\prime \prime}$.

Case 4: $\quad T$ is produced by an operation $\mathcal{O}_{4}$.

Let $\operatorname{sta}(x)=L, \operatorname{sta}(y)=N$ and $y$ is $J_{2}$-avoided. Then $x \in J_{1}$ and, by

Lemma $1, y$ has two neighbors, say $w_{1}, w_{2}$, such that every vertex $w_{i}$ is adjacent to a support vertex. Hence $J_{1} \cup J_{2}$ is a locating kernel of $T$. Let $J^{\prime}$ be a locating kernel of $T$ such that $x \notin J^{\prime}$ and $y \in J_{2}$. Then $N\left(w_{1}\right) \cap J^{\prime}=N\left(w_{2}\right) \cap J^{\prime}=\{y\}$, a contradiction.

Let $J^{\prime \prime}$ be a locating kernel of $T$ such that $x \notin J^{\prime \prime}$ and $y \notin J^{\prime \prime}$. Proving analogously as in Case 1.1.2, we get that there is no such additional locating kernel $J^{\prime \prime}$.

Open Access This article is licensed under a Creative Commons Attribution 4.0 International License, which permits use, sharing, adaptation, distribution and reproduction in any medium or format, as long as you give appropriate credit to the original author(s) and the source, provide a link to the Creative 
Commons licence, and indicate if changes were made. The images or other third party material in this article are included in the article's Creative Commons licence, unless indicated otherwise in a credit line to the material. If material is not included in the article's Creative Commons licence and your intended use is not permitted by statutory regulation or exceeds the permitted use, you will need to obtain permission directly from the copyright holder. To view a copy of this licence, visit http:// creativecommons.org/licenses/by/4.0/.

\section{References}

1. Blidia, M., Chellali, M., Lounes, R., Maffray, F.: Characterizations of trees with unique minimum locating-dominating sets. J. Combin. Math. Combin. Comp. 76, 225-232 (2011)

2. Blidia, M., Favaron, O., Lounes, R.: Locating-domination, 2-domination and independence in trees. Australas. J. Combin. 39, 219-232 (2007)

3. Chellali, M., Mimouni, M., Slater, P.J.: On locating-domination in graphs. Discuss. Math. Graph. Theory 30, 223-235 (2010)

4. Fischermann, M., Volkmann, L.: Unique minimum domination in trees. Australas. J. Combin. 25, 117-124 (2002)

5. Galeana-Sanchez, H., Gomez, R.: $(k, l)$-kernels, $(k, l)$-semikernels, $k$-Grundy functions and duality for state splitting. Discuss. Math. Graph. Theory 27, 359-371 (2007)

6. Haynes, T.W., Henning, M.A.: Trees with unique total dominating sets. Discuss. Math. Graph. Theory 22, 233-246 (2002)

7. Henning, M.A.: Trees with large total domination number. Util. Math. 60, 99-106 (2001)

8. Slater, P.J.: Domination and location in acyclic graphs. Networks 17, 55-64 (1987)

9. Slater, P.J.: Dominating and reference sets in graphs. J. Math. Phys. Sci. 22, 445-455 (1988)

10. Sloane N.J.: The on-line encyclopedia of integer sequences, https://oeis.org/book.html

11. West, D.B.: Introduction to Graph Theory. Prentice-Hall, NJ (1996)

12. Włoch, A.: On 2-dominating kernels in graphs. Australas. J. Combin. 53, 273-284 (2012)

13. Włoch, A., Włoch, I.: On $(k, l)$-kernels in generalized products. Discrete Math. 164, 295-301 (1997)

Publisher's Note Springer Nature remains neutral with regard to jurisdictional claims in published maps and institutional affiliations. 